\title{
Signal characteristics of focal bone marrow lesions in patients with multiple myeloma using whole body T1w-TSE, T2w-STIR and diffusion-weighted imaging with background suppression
}

\author{
Gregor Sommer • Markus Klarhöfer • Claudia Lenz • \\ Klaus Scheffler • Georg Bongartz • Leopold Winter
}

Received: 19 July 2010 / Accepted: 13 August 2010/Published online: 19 September 2010

(C) European Society of Radiology 2010

\begin{abstract}
Objective This study analyses the diagnostic potential of Diffusion-Weighted Imaging with Background Suppression (DWIBS) in the detection of focal bone marrow lesions from multiple myeloma. The signal and contrast properties of DWIBS are evaluated in correlation with the serum concentration of M-component (MC) and compared with established T1- and T2-weighted sequences.

Methods Data from 103 consecutive studies in 81 patients are analysed retrospectively. Signal intensities and apparent Diffusion Coefficients (ADC) of 79 focal lesions in the lumbar spine or pelvis of 38 patients are determined and contrast-to-noise-ratio (CNR) is calculated. Data from patients with low $(<20 \mathrm{~g} / \mathrm{L})$ and high $(>20 \mathrm{~g} / \mathrm{dL}) \mathrm{MC}$ are evaluated separately.

Results Signal intensities of focal myeloma lesions on $\mathrm{T} 2 \mathrm{w}$-STIR vary significantly depending on the MC, which leads to a loss in CNR in patients with high MC. No signal variation is observed for T1w-TSE and DWIBS. The CNR values provided by DWIBS in patients with high MC are slightly higher than those of T2w-STIR. ADC values in patients with low MC are significantly higher than in patients with high MC.
\end{abstract}

\footnotetext{
G. Sommer $(\bowtie) \cdot$ G. Bongartz $\cdot$ L. Winter

Department of Radiology, University of Basel Hospital, Petersgraben 4 ,

CH-4031 Basel, Switzerland

e-mail: gsommer@uhbs.ch

M. Klarhöfer $\cdot$ C. Lenz $\cdot$ K. Scheffler

Department of Radiology, Division of Radiological Physics,

University of Basel Hospital,

Petersgraben 4,
}

CH-4031 Basel, Switzerland
Conclusion Whole-body DWIBS has the potential to improve the conspicuity of focal myeloma lesions and provides additional biological information by ADC quantification.

Keywords Multiple myeloma - Whole-body MRI . Diffusion-weighted MRI · Durie and Salmon plus · Bone marrow

\section{Introduction}

Although conventional radiography is still the standard staging procedure for newly diagnosed and relapsed multiple myeloma (MM) patients in clinical routine, whole-body MRI (WB-MRI) using T1- and T2-weighted contrast-enhanced images has provided evidence of its advantages over conventional skeletal survey [1]. It provides important complementary information as, for example, osteoporosis may be a sign of tumour infiltration, but is difficult to distinguish from senile osteoporosis on conventional radiographs. Also an extramedullary tumour extension could be missed on x-ray images, but is easily detected by means of MRI [2]. Whole-body MRI is therefore recommended at least in MM patients with normal conventional radiography and in all patients with an apparent solitary plasmocytoma of the bone [3]. It must be highlighted that a limited MR examination restricted to spinal and pelvic bone marrow may be inferior to radiographic skeletal survey, whereas wholebody MRI has been demonstrated to be superior to skeletal survey and also to computed tomography $[4,5]$.

There is some evidence that disease burden in patients with MM can even be quantified by MRI. The extent of bone marrow involvement determined by WB-MRI correlates with other conventional parameters of disease and 
may independently predict survival in patients with $\mathrm{MM}$ at the time of initial diagnosis [6].

The key imaging criterion for the staging of multiple myeloma according to the Durie-and-Salmon-plus classification is the number of detectable focal lesions on skeletal survey [7]. With MRI, focal bone marrow involvement is best detected through the use of Turbo Spin-Echo (TSE) Short TI Inversion Recovery (STIR) sequences. Unenhanced T1-weighted SE images are superior in the case of diffuse infiltration, and sensitivity for detecting diffuse infiltration can be increased by signal intensity measurements after contrast administration [8].

Diffusion-weighted imaging with background suppression (DWIBS) was first described by Takahara et al. in 2004 [9]. Since then, various authors have underlined the high potential of the technique in oncological imaging [10, 11]. The inherent contrast of DWIBS - as it is used for the detection of malignant tumour disease-is based on increased signal intensity in tissue with restricted water diffusivity. By this, the technique is capable to detect tissue with increased cellularity non-invasively by measuring the microscopic movement of water molecules [12-14].

Whole-body MRI with diffusion-weighted sequences has been used for staging various solid tumour entities $[10,14,15]$. To date, there are no published studies using DWIBS in the diagnostic work-up of multiple myeloma patients. This study analyses the potential of DWIBS in MM diagnostics by evaluating the bone-marrow-to-lesion-contrast ratio provided by T2w-TSE and DWIBS in correlation with the serum concentration of the M-component (MC).

\section{Materials and methods}

Patients

Data from 103 consecutive studies of 81 patients (48 men, 33 women, mean age 59 years, median age 61 years, age range 25 86 years) were analysed retrospectively. All patients were referred to our department with known or suspected plasma cell disorder. No other acute malignancy was known as indicated by the referring physicians. The patients underwent whole-body MRI with step-wise table motion for initial staging $(n=29)$ or for restaging $(n=74)$. Restaging examinations comprised follow-up studies with prior treatment in 54 cases and without treatment in 20 cases. All procedures were in accordance with the ethical standards of the World Medical Association. The requirements of the local ethics committee were fulfilled.

\section{Imaging protocol}

All examinations were performed on a $1.5 \mathrm{~T}$ whole-body MRI scanner (Magnetom Avanto, Siemens Healthcare.
Erlangen, Germany) using a dedicated 18-channel coil array system (Total imaging matrix (Tim), Siemens Healthcare, Erlangen, Germany). The sequences used were a coronal T1-weighted TSE $(\mathrm{TR}=682 \mathrm{~ms}, \mathrm{TE}=11 \mathrm{~ms}$, matrix size $=384 \times 269$, slice thickness $=5 \mathrm{~mm}$, Field of View $(\mathrm{FoV})=50 \times 50 \mathrm{~cm}^{2}$, acquisition time 1:08 $\mathrm{min}$ ), a coronal T2 weighted STIR $(\mathrm{TR}=9,630 \mathrm{~ms}, \mathrm{TE}=87 \mathrm{~ms}$, $\mathrm{TI}=180 \mathrm{~ms}$, matrix size $=384 \times 269$, slice thickness $=$ $5 \mathrm{~mm}, \mathrm{FoV}=50 \times 50 \mathrm{~cm}^{2}$, acquisition time 2:24 $\mathrm{min}$ ) and a coronal or axial DWIBS (single shot Echo Planar Imaging (ss-EPI), TR $=6,510 \mathrm{~ms}$, TE $=68 \mathrm{~ms}, \mathrm{~b}=0$ and $800 \mathrm{~s} / \mathrm{mm}^{2}$, STIR with fat suppression with $\mathrm{TI}=$ $180 \mathrm{~ms}$, matrix size $=192 \times 96$, slice thickness $=5 \mathrm{~mm}$, FoV $=50 \times 31 \mathrm{~cm}^{2}$, acquisition time 1:43 $\mathrm{min}$ ). The total acquisition time was $30 \mathrm{~min}$. All data were acquired during free breathing. No contrast agent was applied.

\section{Data analysis}

Initial image reading was done as part of the routine work in consensus by two readers with at least 2 years experience in musculoskeletal MRI. Study analysis consisted of signal intensity measurements of the lesions $\left(\mathrm{S}_{\text {les }}\right)$ and of the adjacent bone marrow $\left(\mathrm{S}_{\mathrm{bm}}\right)$ by regions of interest (ROI) in all cases, where focal lesions of the lumbar spine or pelvis were reported, Apparent Diffusion Coefficients (ADC) were determined from automatically calculated ADC maps (linear fit to logarithmical data) using the same ROIs. A maximum of 3 lesions per patient were analysed. Image reading and ROI drawing was done on commercially available workstations (eFilm 2.1.0, Merge Healthcare, Milwaukee, WI, USA). Contrast-to-noise-ratio (CNR) was calculated as

$C N R=\frac{\left|S_{l e s}-S_{b m}\right|}{S D_{\text {noise }}}$

Serum concentrations of the M-component (MC), determined as part of the routine clinical work-up, were used for biochemical correlation. Patients with time differences of more than 5 weeks between the blood test and the MRI examination were excluded. Mean time interval between MRI and MC blood test was 9 days. The patients received no therapy between the blood tests and the MRI examinations.

Statistical analysis

The patients were categorized into two groups based on the $\mathrm{MC}$ levels, group 1 with levels $<20 \mathrm{~g} / \mathrm{L}$, and group 2 with levels $>20 \mathrm{~g} / \mathrm{L}$. Mean values for signal intensities, ADC and CNR were evaluated separately for the two subgroups. A double-sided unpaired $t$-test was used to reveal differences in ADC and CNR between the two subgroups. 


\section{Results}

Seventy-nine lesions in 38 patients were evaluated. 47 lesions were found in 24 patients from group 1 and 32 lesions in 14 patients from group 2. The mean MC from all 38 included patients was $20 \mathrm{~g} / \mathrm{L}$.

Figure 1 shows the results of the signal intensity measurements $\left(\mathrm{S}_{\text {les }}\right)$ : The values provided by the $\mathrm{T} 2 \mathrm{w}-$ STIR sequence vary significantly between the two subgroups yielding lower values in patients from group $1(316 \pm 169)$ compared with group $2(233 \pm 80)(p<0.01)$. No significant difference in $\mathrm{S}_{\text {les }}$ is seen between the two groups for the T1w-TSE (group 1: 271 \pm 74 ; group 2: $254 \pm 75$ ) and the b800-DWIBS $(59 \pm 36 ; 63 \pm 22)$.

The results of the CNR calculations are shown in Fig. 2: According to the course of the signal intensities, significantly higher CNR values are observed in the T2wSTIR images from group $1(79 \pm 66)$ compared with those from group $2(34 \pm 22)(p<0.01)$. Again, no significant variation is seen for the CNR values of the b800-DWIBS $(35 \pm 32 ; 40 \pm 20)$. When comparing the three sequences with respect to their CNR, it is seen that the CNR values provided by the T2w-STIR are significantly higher in patients from group 1 compared with the two other sequences $(p<0.01)$. In patients from group 2 , however, DWIBS provides equal to higher CNR values compared with T2w-STIR. The CNR values generated by the T1wTSE are generally far lower than those of the two other sequences, but nevertheless show a significant small variation between the two groups $(8.2 \pm 7.1 ; 4.1 \pm 3.3)$ $(p<0.05)$.

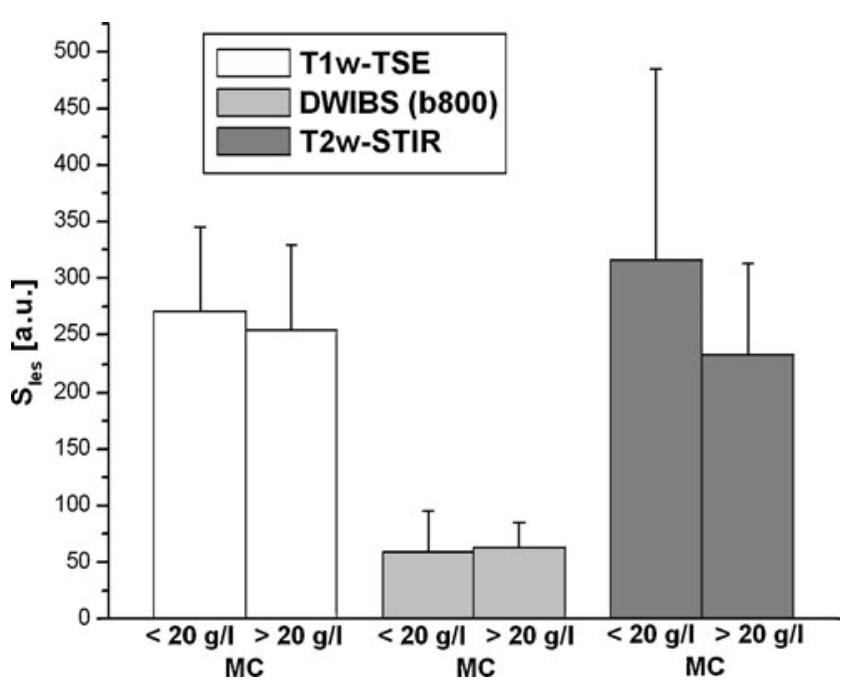

Fig. 1 Measured signal intensities of focal lesions and standard deviations depending on the serum concentration of the M-component (MC). A significant difference in T2w-signal is seen between patients with high and low MC $(p<0.01)$



Fig. 2 Contrast-to-noise-ratios of focal lesions and standard deviations calculated depending on the serum concentration of the $\mathrm{M}$ component (MC). Significant differences between the two subgroups are seen for T2w-STIR and T1w-SE that both provide less CNR in patients with high MC $(p<0.01)$

The results of the ADC measurements are displayed in Fig. 3: Along with the T2w-STIR signal intensities, the ADC also varies significantly with the MC showing higher values in patients from group $1\left([1.37 \pm 0.70] \times 10^{-3} \mathrm{~mm}^{2} / \mathrm{s}\right)$ than in patients from group $2\left([1.00 \pm 0.30] \times 10^{-3} \mathrm{~mm}^{2} / \mathrm{s}\right) \quad(p<0.01)$.

The images in Fig. 4 show signal alterations in a $67 y$ male receiving chemotherapy followed by autologous stem cell transplant. Figure $4 \mathrm{a}-\mathrm{d}$ are the initial MR imaging work up with $\mathrm{MC}$ of $46.8 \mathrm{~g} / \mathrm{L}$ and $80-90 \%$ plasma cell infiltration in bone marrow biopsy. Figure $4 \mathrm{e}-\mathrm{h}$ are the

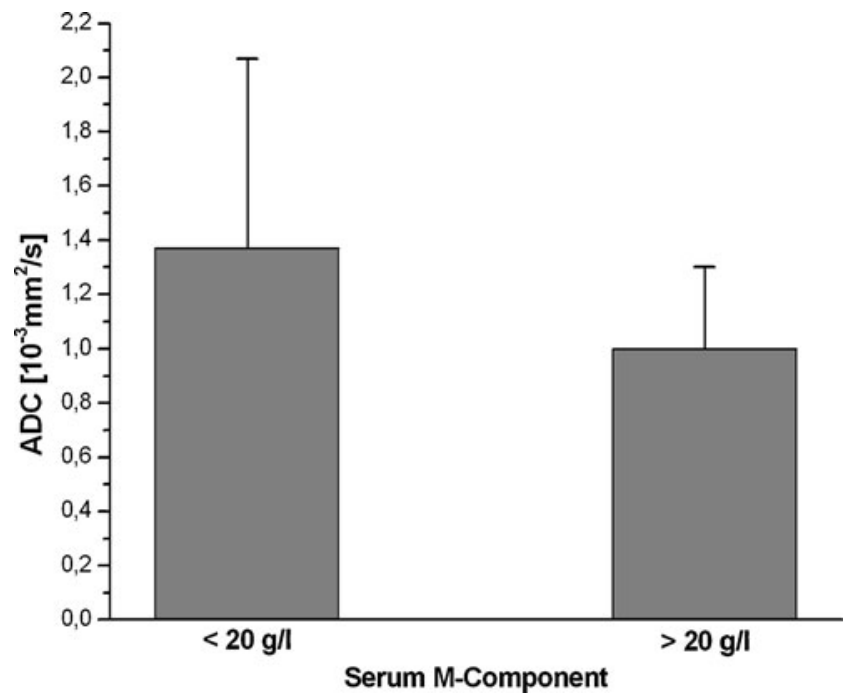

Fig. 3 Mean ADCs of focal lesions and standard deviations depending on the serum concentration of the M-component (MC). A significant difference in ADC is seen between patients with high and low MC $(p<0.01)$ 


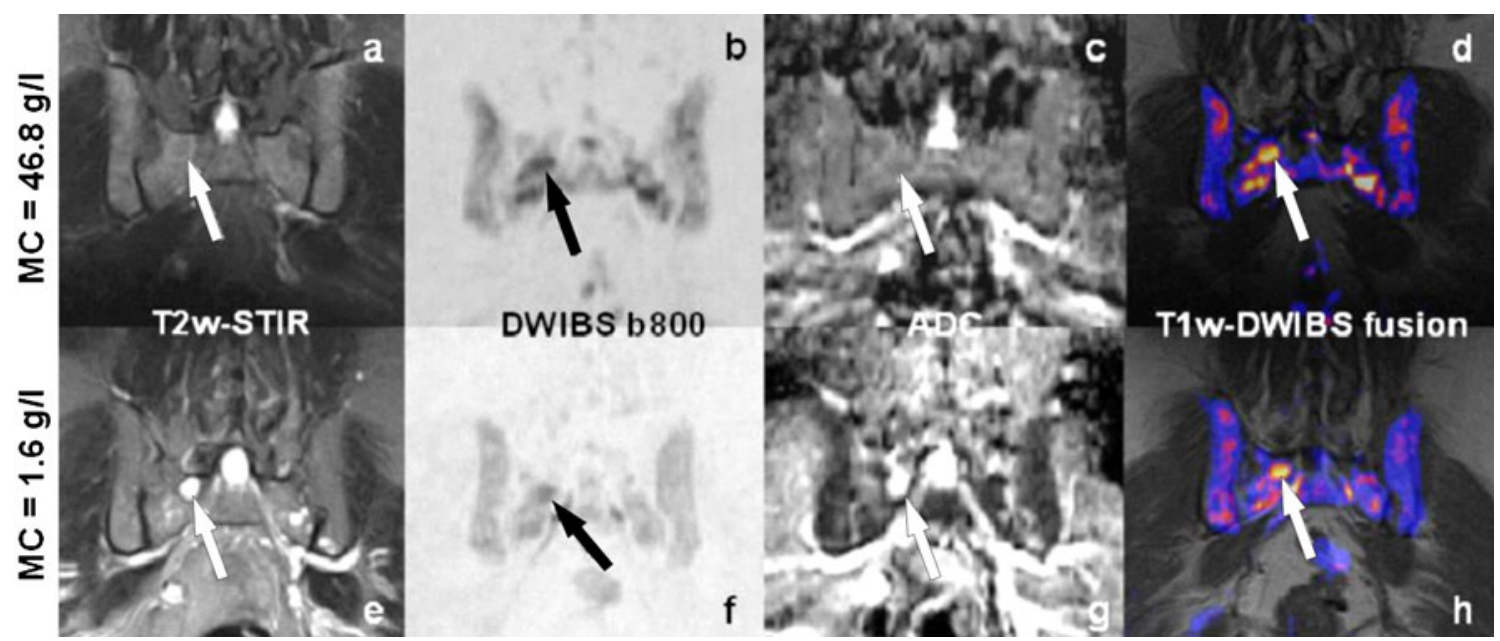

Fig. 4 Sample case of a 67-year-old man who received MR imaging at initial diagnosis (a-d) and 10 months later, after having been treated by chemotherapy and autological stem cell transplantation $(\mathbf{e}-\mathbf{h})$. Note the differences in T2-STIR signal behaviour and ADC at both time points

follow up images 10 months after initial work up and 2 months after stem cell transplant with $\mathrm{MC}$ of $1.6 \mathrm{~g} / \mathrm{L}$. Bone marrow biopsy performed 2 days later showed hyperplastic bone marrow without plasma cell infiltration conformable with complete remission. The displayed lesions in the pelvis are clearly visible as hyperintense spots on the b800 DWIBS images (inverted grey scale) at comparable signal intensity at both time points (b) and (f). On the T2w-STIR image, the lesions are hardly visible at the time of the initial examination (a), but appear strongly hyperintense at follow-up (e). The ADC values of the lesions are accordingly low at the time of initial diagnosis (c), when the patient's paraprotein burden is high and increase towards fluid-equivalent values during follow-up (g), indicating a cystic transformation of the initially solid tumour clusters. In Fig. 4( $\mathrm{d}$ and $\mathrm{h}$ ), a combined T1w-TSE and b800-DWIBS fusion image is displayed, showing the colour-coded DWIBS signal merged onto the corresponding T1-weighted image.

Figure 5 shows signal alterations in a 48 year-old man who presented at initial diagnosis (Fig. 5a-d) with a focal lesion in the left iliac bone, an intra spinal lesion originating from the first lumbar vertebra with compression of the conus medullaris, and an $\mathrm{MC}$ of $34 \mathrm{~g} / \mathrm{L}$. Both lesions have an intermediate to bright signal intensity on T2w-STIR (a) and b800-DWIBS (b) at this time point. Bone marrow
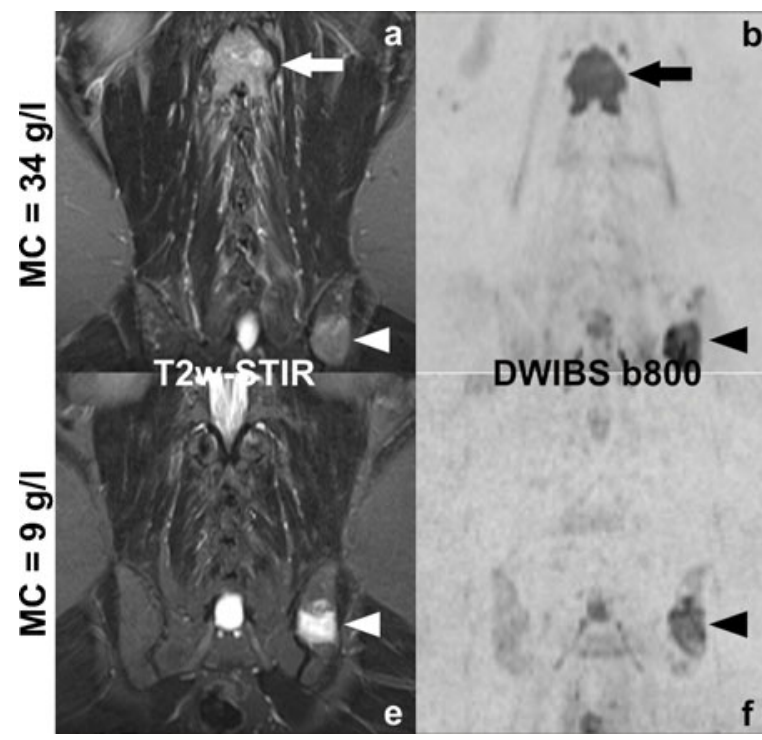

Fig. 5 Sample case of a 48-year-old man who received MR imaging at initial diagnosis (a-d) and 8 months later, before autological stem cell transplantation, after having been treated by chemotherapy $(\mathbf{e}-\mathbf{h})$.

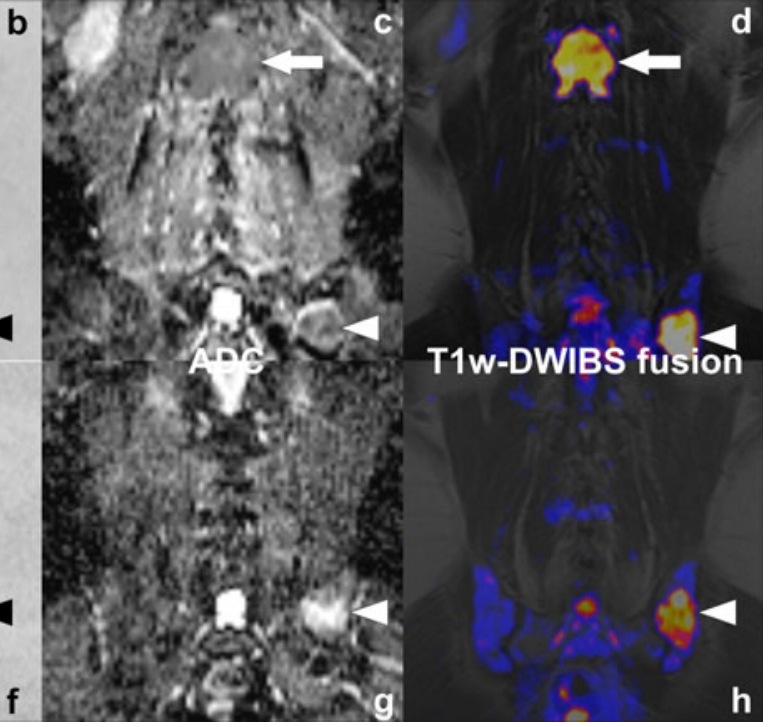

Complete remission of the intra spinal mass (arrows) and an increase in ADC and T2w-STIR signal of the iliac lesion (arrowheads) is seen after therapy 
plasma cell infiltration was found to be $40-50 \%$ in biopsy. Follow up images (Fig. 5e-h) acquired 8 months later after the patient had received systemic chemotherapy and radiotherapy of the lumbar spine show a complete remission of the spinal soft tissue mass and an increase of the ADC of the iliac mass by $\sim 70 \%\left(1.12 \times 10^{-3} \mathrm{~mm}^{2} / \mathrm{s}\right.$ in (c) to $1.94 \times 10^{-3} \mathrm{~mm}^{2} / \mathrm{s}$ in $(\mathrm{g})$ ), accompanied by an increase in T2w-STIR signal (e). MC at this time was $9 \mathrm{~g} / \mathrm{l}$. Bone marrow biopsy performed after consecutive autological stem cell transplant showed complete remission of disease.

\section{Discussion}

This study was performed in order to evaluate the properties of Diffusion-Weighted Imaging with Background Suppression in displaying and characterising focal skeletal involvement in multiple myeloma.

The major result that can be derived from this study is that the signal intensities of focal myeloma lesions in T2wSTIR and the lesion's ADCs are correlated with the serum concentration of the M-component. Based on these findings, we hypothesize that myeloma lesions show different biological characteristics or appearance due to transition from solid lesions of active disease with dense tumor cell clusters and high MC to cystic spongiosa defects filled with fluid or cell detritus and low MC during remission or after a successful treatment. Our hypothesis is supported by the significant change in T2w-STIR signal that is observed between the two groups, indicating a higher amount of fluid in the lesions of patients with low MC. Additionally, lower ADC values are observed in those cases where a high paraprotein burden and thus a high density of (malignant) cells are present. The findings correlate well with the results from previous studies that have evaluated the use of diffusion-weighted MRI with ADC calculation for differentiating benign from malignant vertebral abnormalities, such as a fracture or a mass [16-18].

The change in T2w-STIR signal along with the MC seen in our data leads to the fact that focal lesions tend to be less clearly visible in patients with high paraprotein burden than in patients with low MC which might have a negative influence on the sensitivity of the MRI examination in patients at initial diagnosis or during acute recurrence of disease. Complementary DWIBS imaging may compensate for this, as it provides a high lesion-to-background CNR that is independent from the patient's paraprotein level.

The signal provided by high $b$-value DWIBS showed no significant variation with the MC. This is an unexpected finding following the hypothesis that the lesions transform to cyst-like structures with less restricted water diffusion and thus a decreased DWIBS signal with decreasing MC. The contrast provided by diffusion-weighted spin-echo-EPI sequences at $b$-values of $800 \mathrm{~s} / \mathrm{mm}^{2}$, however, is not exclusively diffusion dependent, but is still sensitive to T2 relaxation effects, known as the T2 shine-through phenomenon. Because of this effect, tissues with long T2 (as fluid) appear more hyperintense compared with those tissues that have equal water diffusivity, but shorter $\mathrm{T} 2$ relaxation times.

Despite the fact that the data evaluated provide statistically significant differences in ADC and T2w-STIR contrast between the two subgroups, the interindividual differences observed in our small patient collective are too pronounced to allow for an accurate determination of the disease burden derived from the individual MRI data. Nevertheless, the data support the assumption that monitoring the lesion's ADCs during follow-up might be useful for estimating disease activity on an intraindividual basis in addition to the clinical parameters. The MC cut-off value of $20 \mathrm{~g} / \mathrm{L}$ that was chosen to separate the two patient groups in our study represents a major criterion for the diagnosis of multiple myeloma as introduced by Durie in 1986 and is since then widely used in clinical practice as a limit for identifying patients that have a considerable paraprotein burden [19]. Also in our patient collective, paraprotein levels $>20 \mathrm{~g} / \mathrm{L}$ were seen predominantly at initial diagnosis or during acute recurrence of disease whereas low levels of the M-component $<20 \mathrm{~g} / \mathrm{L}$ were observed in patients after successful therapy representing a state of remission. Bone marrow biopsy, as indicated in the sample cases in Figs. 4 and 5, may represent a more reliable marker for disease activity in MM. However, due to its invasive character, this technique is less often performed and therefore too little data have been available for a systematic evaluation within this study.

The key imaging criterion for the staging of multiple myeloma according to the Durie-and-Salmon-plus classification is the number of detectable focal lesions [6]. For this reason, only focal lesions were evaluated in this study. We did not correct the results for the effects of slight — and thus invisible - diffuse infiltration of the bone marrow surrounding the focal lesions. According to previously published studies, relevant signal changes in native MRI can be observed only in cases of severe diffuse infiltration of the bone marrow, even if intravenous contrast agent is administered [20, 21]. Patients with a visible combined focal and diffuse pattern of bone marrow infiltration were not respected for signal, contrast and ADC calculations in our study and no significant ADC variation of 'normal' bone marrow between the two subgroups was observed in the patient collective evaluated. The changes in background bone marrow signal that were observed in the T1w-TSE images indicate, nevertheless, that there might be a certain degree of diffuse bone marrow infiltration even though this is not captured on the T2w-STIR and DWIBS images. 


\section{Conclusion}

Whole-body DWIBS imaging is a powerful tool for the staging and follow-up of patients with multiple myeloma. The technique allows for fast and robust whole-body imaging with low technical and operational efforts. Adding DWIBS sequences to whole-body staging protocols can improve the conspicuity of focal MM lesions in patients with newly diagnosed or recurrent disease. Additionally, it provides information on the lesions' biology by ADC quantification. In this way it may serve not only as a tool for lesion detection but also for intraindividual disease monitoring during follow-up of myeloma patients.

Acknowledgements The authors acknowledge Thomas Egelhof MD, Department of Radiology, Merian Iselin Hospital Basel for his fruitful contribution to the conception and initiation of this work.

We also thank Siemens Healthcare for providing customized DWI sequences.

This work was supported by Bayer Schering Pharma, Switzerland. The study sponsor played no role in matters of design, collection, analysis, interpretation of data, and writing the report.

\section{References}

1. Ghanem N, Lohrmann C, Engelhardt M, Pache G, Uhl M, Saueressig U et al (2009) Whole-body MRI in the detection of bone marrow infiltration in patients with plasma cell neoplasms in comparison to the radiological skeletal survey. Eur Radiol 16:1005-1014

2. Dinter DJ, Neff WK, Klaus J, Bohm C, Hastka J, Weiss C et al (2009) Comparison of whole-body MR imaging and conventional $\mathrm{X}$-ray examination in patients with multiple myeloma and implications for therapy. Ann Hematol 88:457-464

3. Dimopoulos M, Terpos E, Comenzo RL, Tosi P, Beksac M, Sezer $O$ et al (2009) International myeloma working group consensus statement and guidelines regarding the current role of imaging techniques in the diagnosis and monitoring of multiple Myeloma. Leukemia 23:1545-1556

4. Lecouvet FE, Malghem J, Michaux L, Maldague B, Ferrant A, Michaux JL et al (1999) Skeletal survey in advanced multiple myeloma: radiographic versus MR imaging survey. Br J Haematol 106:35-39

5. Baur-Melnyk A, Buhmann S, Becker C, Schoenberg SO, Lang N, Bartl R et al (2008) Whole-body MRI versus whole-body MDCT for staging of multiple myeloma. AJR Am J Roentgenol 190:1097-1104

6. Ailawadhi S, Abdelhalim AN, Derby L, Mashtare TL, Miller KC, Wilding GE et al (2010) Multiple myeloma treatment response assessment with whole-body dynamic contrast-enhanced MR imaging. Cancer 254:521-531
7. Durie BGM (2006) The role of anatomic and functional staging in myeloma: description of Durie/Salmon plus staging system. Eur J Cancer 42:1539-1543

8. Baur A, Stabler A, Steinborn M, Schnarkowski P, Pistitsch C, Lamerz R et al (1998) Magnetresonanztomographie beim Plasmozytom: Wertigkeit verschiedener Sequenzen bei diffuser und fokaler Infiltrationsform. Rofo 168:323-329

9. Takahara T, Imai Y, Yamashita T, Yasuda S, Nasu S, Van Cauteren M (2004) Diffusion weighted whole body imaging with background body signal suppression (DWIBS): technical improvement using free breathing, STIR and high resolution 3D display. Radiat Med 22:275-282

10. Sakurada A, Takahara T, Kwee TC, Yamashita T, Nasu S, Horie $\mathrm{T}$ et al (2009) Diagnostic performance of diffusionweighted magnetic resonance imaging in esophageal cancer. Eur Radiol 19:1461-1469

11. Kwee TC, Takahara T, Ochiai R, Katahira K, Van Cauteren M, Imai $\mathrm{Y}$ et al (2009) Whole-body diffusion-weighted magnetic resonance imaging. Eur J Radiol 70:409-417

12. Kwee TC, Takahara T, Ochiai R, Nievelstein RAJ, Luijten PR (2008) Diffusion-weighted whole-body imaging with background body signal suppression (DWIBS): features and potential applications in oncology. Eur Radiol 18:1937-1952

13. Matsumoto Y, Kuroda M, Matsuya R, Kato H, Shibuya K, Oita M et al (2009) In vitro experimental study of the relationship between the apparent diffusion coefficient and changes in cellularity and cell morphology. Oncol Rep 22:641-648

14. Takenaka D, Ohno Y, Matsumoto K, Aoyama N, Onishi Y, Koyama $\mathrm{H}$ et al (2009) Detection of bone metastases in non-small cell lung cancer patients: comparison of whole-body diffusionweighted imaging (DWI), whole-body MR imaging without and with DWI, whole-body FDG-PET/CT, and bone scintigraphy. J Magn Reson Imaging 30:298-308

15. Bohlscheid A, Nuss D, Lieser S, Busch H (2008) Tumorsuche mittels kernspintomografischer Diffusionsbildgebung-Erste Erfahrungen. Rofo 180:302-309

16. Balliu E, Vilanova JC, Pelaez I, Puig J, Remollo S, Barcelo C et al (2009) Diagnostic value of apparent diffusion coefficients to differentiate benign from malignant vertebral bone marrow lesions. Eur J Radiol 69:560-566

17. Pui MH, Mitha A, Rae WID, Corr P (2005) Diffusion-weighted magnetic resonance imaging of spinal infection and malignancy. $\mathrm{J}$ Neuroimaging 15:164-170

18. Raya JG, Dietrich O, Reiser MF, Baur-Melnyk A (2005) Techniques for diffusion-weighted imaging of bone marrow. Eur J Radiol 55:64-73

19. Durie BG (1986) Staging and kinetics of multiple myeloma. Semin Oncol 13:300-309

20. Schmidt GP, Baur A, Stabler A, Schoenberg SO, Steinborn M, Baltin V et al (2005) Beurteilbarkeit diffuser Knochenmarksinfiltrationen der Wirbelsaule bei multiplem Myelom: Korrelation von MRT-Befunden mit der Histologie. Rofo 177:745-750

21. Wasser K, Moehler T, Nosas-Garcia S, Rehm C, Bartl R, Goldschmidt $\mathrm{H}$ et al (2005) Korrelation zwischen MRT und Histopathologie des Knochenmarks bei Patienten mit Multiplem Myelom. Rofo 177:1116-1122 